\title{
Emociones en torno a la violencia hacia las mujeres Drag queen
}

\author{
Emotions around violence against Drag queen women
}

\author{
Alba Luz Robles Mendoza \\ Facultad de Estudios Superiores Iztacala UNAM \\ albpsic@unam.mx \\ https://orcid.org/0000-0003-3778-4083 \\ Danae Soriano Valtierra \\ Facultad de Estudios Superiores Iztacala UNAM \\ danae.soriano@iztacala.unam.mx \\ https://orcid.org/0000-0002-6587-736X
}

\section{Resumen}

Introducción: La violencia hacia mujeres es una manifestación de desigualdad y poder entre géneros, reforzado socioculturalmente por estereotipos sexuales; trascendiendo hacia nuevas expresiones de las sexualidades diversas, pasando de una violencia estructural a una violencia basada en el género y de violación de derechos humanos. Objetivo: Conocer las emociones relacionadas con la violencia que sufren las personas Drag Queen en su cotidianeidad. Metodología: Se hicieron entrevistas semiestructuradas a tres Drag Queen, elegidas a través del método bola de nieve. Se analizaron los discursos desde una perspectiva de género y de diversidad sexual. Resultados. La principal emoción reportada es el miedo a ser golpeadas y el enojo a no poder evitarlo. Mencionan que adoptan conductas estereotipadas al género femenino, quedando impotentes para contestar desde lo masculino. Buscan el apoyo social y si están solas analizan si pueden defenderse como hombres o huir como mujeres, evitando ser lastimadas o asesinadas (crimen de odio). Conclusiones. Las emociones sobre violencia experimentada por las Drag Queen no varían de las expresadas por mujeres Cis, ya que siguen siendo desigualdades de poder y de género. Se requiere de políticas públicas en pro del derecho al libre ejercicio de las sexualidades en las expresiones de género.

Palabras clave: Drag Queen; género; violencia; emociones. 


\begin{abstract}
Introduction. Violence against women is a manifestation of inequality and power between genders, socio-culturally reinforced by sexual stereotypes; transcending towards new expressions of diverse sexualities, going from structural violence to gender-based violence and violation of human rights. Objective. To know the emotions related to violence suffered by Drag Queen people in their daily lives. Method. Semi-structured interviews were conducted with three Drag Queens, chosen through the snowball method. The discourses were analyzed from a gender and sexual diversity perspective. Results. The main emotion reported is fear of being beaten and anger at not being able to avoid it. They mention that they adopt stereotypical behaviors to the feminine gender, being powerless to respond from the masculine. They seek social support and if they are alone, they analyze whether they can be able to defend themselves as men or flee as women, avoiding being hurt or killed (hate crimes). Conclusions. The emotions about violence experienced by Drag Queens do not vary from those expressed by Cis women, as they continue to be inequalities of power and gender. Public policies are required in favor of the right to the free practice of sexualities in gender expressions.
\end{abstract}

Key words: Drag Queen; gender; violence; emotions. 


\section{Introducción}

Se entiende a la violencia hacia las mujeres como todo acto de fuerza física, verbal o psicológica, dirigida a la mujer o niña, que le cause daño físico o psicológico, humillación o privación arbitraria de su libertad y que perpetúe la subordinación femenina, tanto si se producen en la vida pública como privada. La violencia hacia las mujeres es una de las manifestaciones más claras de la desigualdad de poder de los hombres sobre las mujeres, reforzado socioculturalmente por los estereotipos sexuales exacerbados, trascendiendo hacia la expresión de lo femenino y lo masculino dentro de la cotidianeidad. De ahí su importancia para su estudio, en las nuevas expresiones de las sexualidades diversas. La extensión de nuevas transformaciones de lo femenino (transexuales, travestis, transgénero, dragqueenismo, entre otras) ha permitido que la violencia hacia las mujeres se amplíe hacia cualquier persona o grupo de personas sobre la base de su orientación o identidad sexual, sexo o género, que impacta de manera negativa en su identidad y bienestar social, pasando de ser una violencia de género entre hombres y mujeres a otro tipo de violencia estructural a la que llamaremos violencia basada en el género, referida a las expresiones de identidades sexuales diversas no binarias. Es decir, las víctimas sufren violencia por el solo hecho de expresarse como mujer, siendo un problema de derechos humanos más que de sexos.

\section{Antecedentes teóricos}

Son dos las realidades fundamentales que caracterizan la experiencia de las personas en la vida social. Una es la simbólica, realizada principalmente a través del uso del lenguaje verbal y otra es la física, situada en lo corporal y en la representación social de ésta; haciendo al cuerpo partícipe de los procesos de objetividad-subjetividad y de socialización de la cultura en la cual se encuentra inmerso el ser humano. Ambas realidades interactúan constantemente como procesos relacionales duales en la construcción de la identidad del individuo, transformándolos en actores sociales inscritos en una cultura específica (Salinas, 1994).

Las personas dan sentido a su existencia e inteligibilidad a sus cuerpos, la cual se basa en la condición de género del sujeto. Así, se nace con un cuerpo que posee vulva y clítoris e internamente vagina, y se considera socialmente una mujer, partiendo de estos genitales que indican su sexo como categoría de hembra. A una persona cuyo cuerpo anatómico posee escroto y pene como órganos genitales externos se le considerará socialmente un hombre, y sexualmente un macho o varón. Recalde y Gómez (2016:41) hacen mención a estas personas como cisgénero ${ }^{1}$, es decir que tienen la identidad de género que les fue asignada al nacer según la morfología de sus órganos genitales. Maffia y Cabral (2003:86) sostienen que el sexo anatómico y su propia dicotomía son producto de una lectura ideológica de género, que nos permite hablar de un sexo natural y suficientemente fuerte para disciplinar a los cuerpos cuando no se adaptan cómodamente a la lectura social de lo que se espera de ellos. Es decir, si pensáramos en la materialidad del cuerpo, no podríamos entenderla fuera del lenguaje del género. Recalde y Gómez (2016:42) plantean esta materialidad vinculada con las características fenotípicas y anatómicas, que hacen que las particularidades se entiendan bajo la categoría de "sexo".

\footnotetext{
${ }^{1}$ Cisgénero es un término que surge de los colectivos transgéneros, ya que tanto "cis" como "trans" son prefijos del latín que indican de un lado y al otro lado respectivamente. En este sentido, indicaría estar del lado del género -ser ciso al otro lado del género -ser trans-.
}

Revista del Laboratorio Iberoamericano para el Estudio https://doi.org/10.46661/relies.6288

Sociohistórico de las Sexualidades 
Por otro lado, Lamas (2016:156) nos invita a reflexionar sobre las acepciones del concepto género, descrita como una simbolización cultural, que revela una lógica presente en todas las dimensiones de la vida social, que condiciona las normas sociales y el sistema jurídico, y tiñe la construcción de la identidad psíquica de las personas.

Desde esta perspectiva, Stoller (1968, citado en Lamas 2016:158) menciona que la categoría género se articula en 3 instancias básicas: 1) La asignación de género, entendida como la atribución que se establece a partir de la apariencia externa genital, 2) la identidad de género, que se determina durante el desarrollo de acuerdo con cada código cultural y, 3) el rol de género, que se forma con el conjunto de normas y prescripciones que transmite la sociedad sobre el comportamiento femenino o masculino.

De forma similar, Rubín (1975, citado en Lamas, 2016:159) define al sistema sexo/género como el conjunto de acuerdos a partir de los cuales una sociedad transforma la sexuación en un producto de la actividad simbolizada de los seres humanos, por lo que cada sociedad establece un conjunto de normas a partir de las cuales el sexo y la procreación son moldeadas por la intervención social, y se satisface de una manera "natural".

Desde otra mirada, Scott (1997, citada en Lamas, 2016:159) consolida la definición de género como una forma primaria de relaciones significantes de poder, donde se permite que hombres y mujeres sean los pilares de un sistema fundamentado en un orden simbólico jerarquizado de poder $y$ dominación masculina.

Ante estas concepciones, podríamos plantearnos la siguiente pregunta: ¿Existirá el género como categoría social sin sexo? En 1990, Judith Butler viene a dar un giro a los conceptos tradicionales del género, planteando que el género resulta ser performativo, es decir, que constituye la identidad que se supone que es. Butler (1993:17) toma distancia de la diferencia ya conocida entre sexo y género y nos replantea al género como un acto transformador donde se va y viene dentro de las categorías que lo sustentan. Su principal aportación se origina en la convicción de que, "el sujeto social se produce a través de medios lingüísticos" (Butler,1997; citado en Moreno y Torres, 2018:241).

Para Butler (2001:17) el performativo es un dominio en donde el poder actúa como discurso, funciona como un dispositivo en la configuración del género al imponer una marca lingüística, una denominación interpelada sobre los cuerpos. Esta marca, inicia su proceso de feminización o masculinización, no de forma natural, -no hay una esencia biológica que le dicte su ley- sino artificial, convencional.

Desde este punto de vista, Moreno y Torres (2018:243) infieren que Butler rompe con la interpretación constructivista del género al refutar la existencia del sexo como un sustrato biológico sobre el cual se construye culturalmente el género, desde el momento de la etiquetación de la persona como "niño o niña" se inicia una serie de actos rituales, convencionales, institucionales, que compelen al referente-sujeto "niño o niña", al actuar de acuerdo a las normas del género, crea el género en su propia actuación. Los actos performativos también tienen un doble anclaje referencial; son a la vez su propio acontecimiento, la irrepetible identidad de un cuerpo.

Para Butler (2001:266) los movimientos, gestos, ademanes y reacciones corporales son signos mediante los cuales se expresa una autoridad convencional, un mandato previo que determina al 
sujeto en tanto dicción social. La convención social precedente al ordenamiento de género es la heterosexualidad, el horizonte normativo del género es la matriz heterosexual².

En este sentido, la orientación sexual designa la dirección de los deseos amorosos, románticos o eróticos, sin interferir con la identidad de género. El sexo, el género y la orientación sexual, son los tres criterios binarios de los que hoy por hoy, se hace uso y sirven a los sistemas jurídicos para definir y clasificar las identidades sexuales (Bernini, 2017:51).

El sexo biológico (macho-hembra), el sexo social (hombre-mujer), el género (masculino-femenino) y la orientación sexual (heterosexual-homosexual o bisexual) no son suficientes para dar cuenta de la complejidad de las construcciones corpóreas y de las identidades sexuales y de género de los seres humanos.

El binarismo sexual, que reduce la complejidad de los factores que intervienen en la definición de la identidad sexual de los seres humanos imponiendo rígidas alternativas al sexo, género y orientación sexual, resultan ser vectores de poder que hacen inteligibles las expresiones de la sexualidad, así como también aquellas identidades que se obtienen por composición de estos tres criterios (Bernini, 2017:60).

Dicho binarismo deja fuera las identidades y orientaciones disidentes. Córdova (2011:47), hace referencia a que en las sociedades occidentalizadas existe una relación mimética entre sexo, género, sexualidad y deseo, de forma que existe una inteligibilidad unívoca al interior del sistema binario sexo-género.

Sin embargo, existe otro antecedente, planteado por Bernini (2017:64) ante el diagnóstico de la homosexualidad y transexualidad, planteado en el DSM (Manual Diagnóstico y Estadístico de Desórdenes Mentales), redactado por la Asociación Americana de Psiquiatría, donde el término de homosexualidad egosintónica ${ }^{3}$ apareció hasta 1987, y de homosexualidad egodistónica ${ }^{4}$ hasta 1990 ; aunque el transgenerismo, todavía está presente en la tercera edición del DSM de 1980. En la cuarta edición de 1994, cambia su conceptualización a transexualismo o trastorno de la identidad de género. Para la quinta edición publicada en el 2013 hasta nuestros días, ha sido etiquetado como "disforia de género".

Pérez (2011) y Bernini (2017) aluden a la identidad transgénero, como una identidad política que desafía al binarismo sexual y que promueve la transformación y la define como postransexual, porque cuestiona el modo en que la transexualidad ha sido concebida, según la lógica del binarismo y su difusión ha despertado la conciencia de movimientos LGBTTTIQ+ de todo el mundo.

El término transexual parece por primera vez en un artículo publicado por David Caldwell en 1949 llamado "Psychopathia Transeualis" pero es a partir de 1960 cuando se desarrolla el concepto de transexual, con un cambio de paradigma en las prácticas de lo trans, debido a la introducción de tecnologías de modificación corporal, como las terapias hormonales y las cirugías de reasignación sexual. El concepto transgénero data de la década de 70's, por Virginia Prince, quien lo definía como una tercera vía entre la transexualidad y el travestismo, y se definía como vivir plenamente en el género contrario al asignado al nacimiento, sin necesidad de recurrir a lo que la medicina llama

\footnotetext{
${ }^{2}$ La matriz heterosexual establece el marco binario donde los sujetos se hacen inteligibles mediante la incorporación de ciertos ideales de feminidad y masculinidad, ideales que casi siempre se relacionan con el vínculo heterosexual.

${ }^{3}$ Homosexualidad egosintónica: la de aquellos sujetos que aceptan su deseo homosexual como una parte de sí mismos que se integra armoniosamente con su propia personalidad.

${ }^{4}$ Homosexualidad egodistónica: la de aquellos sujetos que viven su deseo homosexual con malestar.
}

Revista del Laboratorio Iberoamericano para el Estudio Sociohistórico de las Sexualidades https://doi.org/10.46661/relies.6288 
"cirugías de reasignación genital o sexual", usando este concepto para autonombrarse (Pons y Garosi, 2016:310).

El concepto transgénero se convirtió en una especie de sombrilla, que acogió la pluralidad de experiencias que cuestionaban la coherencia, estabilidad y correspondencia entre género, cuerpo y deseo, donde se sustentaba el binarismo de género, significado cultural para la matriz heterosexual (Pérez, 2011:99; Pons y Garosi, 2016:314).

El travestismo, por otro lado, ha sido considerado como una forma de fetichismo, donde la ropa es el objeto sexuado, siendo así un tipo de parafilia. En el travestismo se hace referencia a vestirse con ropa del sexo opuesto, siendo más frecuente este tipo de prácticas por los hombres. Sin embargo, no desean cambiar de sexo, como en el caso de los transexuales. Además, la mayoría de ellos no tienen un sentido interno de pertenecer al sexo opuesto, como les ocurre a las personas con disforia de género (Cepeda y Flores, 2011:18).

Similarmente, encontramos a las Drags Queens, o imitadoras femeninas, quienes se diferencian de las personas transexuales y travestis, en el sentido de que son individuos que visten ropa femenina con el objetivo explícito de actuar frente al público (Schacht, 2000, citado en Moncrieff \& Lienard, 2017:2).

Por muchos años en la historia del teatro, desde la época clásica de Grecia y Roma hasta el teatro de Shakespeare en el siglo XVII, se tuvo como principal prohibición la intervención de mujeres en la actuación, por lo que se optaba en travestir a actores varones para que realizaran caracterizaciones femeninas. Se cree que de este contexto surge el nombre "Drag Queen", por un lado, el término "Drag" es una palabra formada por la abreviación (clipping) de la frase dress like a girl o dress roughly as girls; donde hombres se vestían como mujeres para actuar y, por otro lado, "Queen" sugiere que los personajes a caracterizar hayan sido, en su mayoría, relativos a la realeza, de esa manera las Drag Queen se fueron desarrollando en el ámbito teatral. Hacia los años cuarenta, en Estados Unidos, se convocaban en bares a comediantes y animadores para que representarán papeles de mujeres y, en los años setenta, el Drag se masifica con el boom de la música disco (ORÉ, 2009, citado en Cepeda y Flores, 2011:18).

También, se encuentra la base de la lexicografía, donde, según el Dictionary of Slang and Unconventional English, de Eric Partridge, la palabra Drag se refiere a las enaguas que utilizaban las mujeres a partir de mediados del siglo XIX en Inglaterra y Estados Unidos. Su efecto en el movimiento de las mujeres era similar al de los frenos de los trenes o carrozas al ralentizarlos. Dicha prenda no sólo formó parte de los atavíos de las mujeres, sino también de los hombres que actuaban como mujeres en los teatros y de los hombres homosexuales (Ilamados mollies) que asistían a reuniones privadas vestidos como mujeres (Rupp \& Taylor, citados en Villanueva, 2017:97).

Existen diferentes teorías acerca del surgimiento del término Drag, sin embargo, la más aceptada se atribuye a la descrita a mitad del siglo XIX en Oriente, donde se llevaban a cabo representaciones teatrales conocidas como "Burlesque victoriano". Posteriormente, en ese mismo siglo, los habitantes de Gran Bretaña vivían una particular época de esparcimiento, con nuevas formas de entretenimiento que se hacían más populares a través del teatro, el cual tenía un gran impacto social como en la actualidad lo tiene la televisión y el internet. Estos eventos iban acompañados de música y connotaciones políticas y sociales a través del uso de la comedia, la parodia y los estereotipos socioculturales (Victoria, 2015; citado en Toquero, 2018:4). 
En esa época, de acuerdo con Bett (2004, citado en Toquero, 2015:12) se utilizaba el cross-dressing, que significa utilizar ropa y comportamiento considerado por una cultura determinada por ser apropiada para otro género, de allí también se plantea el empoderamiento del término Drag, a través de la puesta en escena donde se ve reflejada la teoría Queer.

Desde la perspectiva de Cepeda y Flores (2011) es usual que se tienda a reducir la performance Drag a la representación de mujeres, sin embargo, hace una puntual referencia a la reflexión de RuPaul, una famosa Drag Queen estadounidense, quien comenta que él no se personifica de mujer “¿Cuántas mujeres usan tacones de siete pulgadas, pelucas de cuatro pies y vestidos ajustados?" "Yo no me visto como mujer me visto como una Drag Queen" (pág. 18). A partir de esta reflexión, se establece la relevancia de entender que una Drag Queen es, necesariamente, un hombre que se escenifica de manera exagerada, nunca pudiendo ser performado por una mujer. Una Drag Queen se viste, maquilla y prepara estéticamente para realizar un show que es considerado su trabajo. En ese sentido, es una artista que, por lo general, realiza desde mímica, baile, shows de comedia, hasta performances conceptuales.

Ser y vivir como drags es tratar de romper esos estereotipos puestos por la sociedad, manejan un nuevo lenguaje y formas particulares de hablar, esto se ve reflejado a través de su vestuario, maquillaje y forma de actuar. Las drags actúan y hacen representaciones escénicas que pueden ser mejor conocidas como exhibiciones, en donde buscan llamar la atención y causar emociones en los espectadores, aunque en ocasiones pueden transformarse por gusto o comodidad, el fin siempre será emitir una reacción por medio de una audiencia, ya que sin ella su existencia no tendría ningún sentido (Toquero, 2018).

La performatividad en las drags se hace explícita cuando apreciamos el desenvolvimiento del personaje dentro del show. Asimismo, juegan con múltiples personalidades que sobrepasan lo masculino y lo femenino, un caso representativo es el de Nébulah-una reconocida Drag Queen de la ciudad de México-, quien no busca cubrir sus rasgos masculinos; sino que, por el contrario, los exhibe y lo utiliza como distintivo en sus presentaciones (Strubel-Scheiner 2011:13).

Las Drag Queens son "seres liminales", donde la "liminalidad" se desarrolla en el texto de la antropóloga Mary Douglas, titulado "Pureza y peligro", haciendo referencia al sujeto inclasificable dentro de las categorías cerradas que determina el discurso de género heteronormativo. Según Douglas, el desorden contiene poder en sí mismo, pues, al encontrarse más allá de las fronteras y clasificaciones construidas socialmente sobre lo que es o no viable, se trata de un espacio de posibilidades infinitas de creación y recreación de nuevas actuaciones; además, juega con conceptos como "peligro" pues al escapar del orden social, coloca en evidencia los frágiles límites de la cultura (Cepeda y Flores, 2011: 20). 
Figura 1. Drag Queen Ábora Nuit

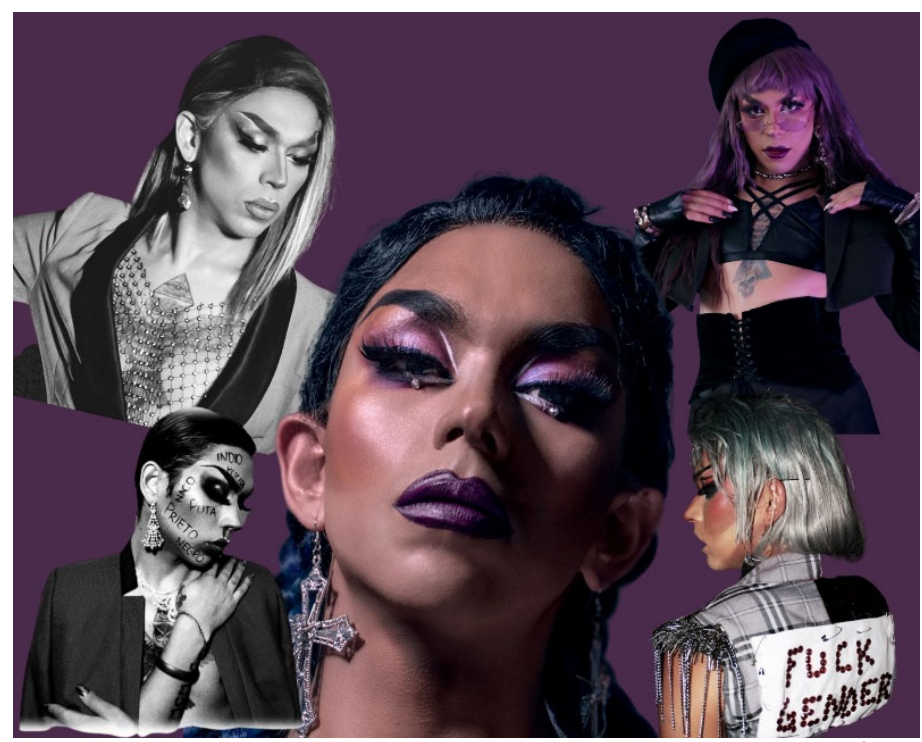

Collage realizado con fotografías del Instagram de la Drag Queen Ábora Nuit Fuente: https://instagram.com/abora.nuit?utm_medium=copy_link

De esa manera, es posible señalar que la Drag Queen posee un poder especial, que se manifiesta en los sentimientos que genera en su audiencia, los cuales pueden ser alegría, atracción, intimidación, miedo, entre otros. Al trasgredir los órdenes de género, la Drag Queen es percibida como una crítica al sistema performativo de género o lo que Frau Diamanda denomina "terrorismo del género" (Cepeda y Flores, 2011:20).

A partir de los reality shows, los estudios coinciden en que la televisión ha sido utilizada para reforzar construcciones de identidad, lo que permite el empoderamiento de las drags dentro de las formas lingüísticas y culturales de los medios sociales (Prince, 2010, citado en Toquero, 2018:10).

Cepeda y Flores (2011) plantean que el movimiento Drag quiebra, cuestiona y relativiza este discurso a través de sus performances. Las Drag Queens juegan con las nociones y las estéticas de ambos géneros para construirse a sí mismas de forma híbrida, son artistas que transgreden las dicotomías, pero cuya identidad no radica en el género sino en su misma construcción como una imagen o mensaje estético y también como un "producto" que puede mercantilizarse y significar una oportunidad de trabajo e ingresos económicos.

En defensa de una definición del cuerpo femenino como una realidad cultural en lugar de biológica esencial, se contempla a un cuerpo cambiante al entrar en contacto con el entorno social, siendo sujeto a significados diversos, importantes para la interacción social. En este momento, la esencialidad biológica pasa a un segundo plano, frente a la acción de la cultura social, creando y modificando nuevas realidades (Salinas, 1994).

La extensión de nuevas transformaciones de lo femenino (transexuales, travestis, transgénero, Drag Queen) han permitido que la violencia dirigida hacia las mujeres se amplíe hacia cualquier persona o grupo de personas sobre la base de su orientación, identidad sexual, condición sexual o de género, que impacta de manera negativa en su bienestar biopsicosocial, pasando de ser una violencia de género hegemónica patriarcal donde prevalece la dominación masculina frente a la femenina a una violencia basada en el género caracterizada por la supremacía del hombre hacia cualquier persona fuera de la categoría binomial hombre-mujer y que se encuentra en condiciones de vulnerabilidad social frente a dicha posición dominante. Los factores que interactúan en la construcción de la 
identidad sexual femenina, a la que hacemos referencia, pertenecen a una realidad social que se manifiestan desde esta violencia estructural, llamada violencia de género y a la vez desde la vulnerabilidad social en la que la comunidad LGBTTTI se ubica como grupo minoritario, llamada violencia basada en el género (VBG). Es decir, las víctimas sufren violencia no solo por el hecho de expresarse socialmente como mujeres, sino además frente a una violación de derechos humanos y de vulnerabilidad social, política y cultural.

Martínez (2013, citado en CNCLVD, 2014) en su estudio cualitativo sobre el "Tratamiento de la violencia basada en el género en medios de comunicación y fuentes informativas" define a la VBG como una categoría de análisis que aporta un marco normativo para un conjunto de violencias de orden de género determinado por jerarquías de poder, desigualdad, discriminación e inequidad social. En este sentido, la VBG afecta desde niños, niños y adolescentes que viven o presencian violencia familiar hasta hombres que no cumplen con el mandato de género o personas que no se rigen por la sexualidad heteronormativa.

Retomando a Galtung (2003, citado en Acién y Checa, 2020), la violencia basada en el género se caracteriza por su esencia estructural, cultural y directa, justificándose en las prácticas sociales y en la expresión de éstas dentro de los contextos donde se encuentran inmersos las personas. La vulneración de los derechos humanos que pueden tener las nuevas expresiones de mujeres dentro del colectivo LGBTTTI, coloca al Estado y a sus instituciones frente a las discriminaciones que conlleva la violencia basada en el género y que limita el ejercicio y goce de la sexualidad diversa.

Acién y Checa (2020) confirman que la violencia cultural legitima la violencia directa y estructural, donde las mayorías sociales arraigan las normas y valores a lo preestablecido, sosteniendo el binarismo, la desigualdad y la violencia hacia las mujeres y las expresiones femeninas.

Desde este paradigma, el objetivo de este trabajo fue conocer las emociones relacionadas con la violencia que sufren las personas Drag Queen que se expresan como mujeres en el ámbito artístico, haciendo un análisis desde el enfoque de género de las expresiones de la violencia basada en el género hacia los grupos sociales minoritarios y de mayor vulnerabilidad social.

\section{Precisiones Teórico-Metodológicas}

Esta investigación se realizó a través de una metodología de tipo cualitativa, siendo un estudio exploratorio y descriptivo. La importancia en la elección del diseño cualitativo recae en el hecho de que estas investigaciones permiten conocer la realidad de un fenómeno determinado a través de los significados o ideas que expresan los participantes, es decir, el conocimiento se construirá a partir del discurso, experiencias y percepciones de éstos (Hernández, et al, 2018).

Para la obtención de la información se realizaron y grabaron entrevistas semiestructuradas, dado que el objetivo de las mismas fue obtener información y acceder al fenómeno de estudio a través del lenguaje, expresiones, ideas y creencias de las participantes. Los criterios para la selección de las informantes fueron: desarrollarse en el medio artístico como Drag Queen, estar interesadas en participar y haber proporcionado su consentimiento informado una vez leído, entendido y aceptado su participación en la investigación. Se hicieron tres entrevistas, las cuales constaban de 4 partes. En la primera parte se preguntaba sobre la inserción en la práctica artística del Drag Queen, cómo la conocieron y qué significa para ellas el género. Posteriormente, en la segunda parte se preguntaba sobre la primera vez que iniciaron a vestirse y actuar como drag, cómo surge su nombre artístico y qué significa para ellas esta expresión sexual femenina. La tercera parte de la entrevista se involucra el tema de la violencia de género, la violencia que sufren como expresiones de mujer en el ámbito 
artístico, las discriminaciones a las que se enfrentan y cómo viven sus emociones desde esta violencia, finalizando con la última parte que constituye el cierre de la entrevista sobre sus planes futuros en el Dragqueenismo. Las entrevistas duraron aproximadamente dos horas y fueron grabadas y realizadas en sitios que representaran comodidad e intimidad para las participantes. Dos de ellas se llevaron a cabo en los hogares de las entrevistadas y la última fue en una sala de docentes de la Universidad donde estudiaba.

La obtención de las participantes se realizó a través del muestreo por bola de nieve, el cual consiste en iniciar el estudio con una informante, a través del cual se obtienen los datos y participación de una más, mediante un sistema en cadena, el cual se repetirá con las siguientes entrevistadas. Este método fue el más óptimo, dado que es difícil acceder a ellas de forma directa sin sentir que pueden ser afectadas en su vida personal.

Una vez obtenida la información se realizó la categorización con base en la propuesta de análisis de datos de Richard Krueger, la cual incluye: 1) Obtención de datos, mediante la realización de entrevistas semiestructuradas, 2) Manejo de la información, por medio de las transcripciones de las entrevistas grabadas, 3) Codificación, a través de la lectura crítica de las entrevistas, se identificaron los temas principales y relevantes, permitiendo la clasificación en códigos, categorías y subcategorías, y 4) Verificación, una vez analizada la información fue pertinente contrastar y corroborar la información con las informantes (citado en Alvarez-Gayou, 2006). El análisis de la información de las entrevistas se fundamentará a través de la perspectiva de género y de diversidad sexual.

Uno de los criterios fundamentales de la investigación fue actuar bajo los principios éticos básicos como medida para proteger y asegurar los derechos de las participantes. Por tanto, esta investigación se basó en los principios del informe de Belmont ${ }^{5}$, marcando como prioridad el respeto por las personas, a las cuales se les protegió su autonomía al cuidar la voluntariedad en su participación, así como la libertad de permanencia o abandono; para esto se le otorgó un consentimiento informado individual previo a la entrevista.

\section{Hallazgos y discusión de los mismos}

Las participantes que intervinieron en esta investigación fueron tres. A continuación, en la siguiente tabla se describe su perfil. Los nombres reales fueron cambiados por nombres de galaxias para garantizar la confidencialidad.

Tabla 1. Participantes del estudio

\begin{tabular}{|l|c|c|c|}
\hline \multicolumn{1}{|c|}{ NOMBRE } & SEXO & EDAD & OCUPACIÓN \\
\hline Andrómeda & Hombre & 29 & Drag Queen en clubs nocturnos \\
\hline Hydra & Hombre & 25 & Drag Queen en clubs nocturnos \\
\hline Columba & Hombre & 24 & Estudiante de la Licenciatura en Psicología \\
\hline
\end{tabular}

Se presentan los datos generales de las participantes. Fuente: Autoría propia

${ }^{5}$ CONBIOÉTICA. Informe Belmont. Principios y guías éticos para la protección de los sujetos humano de investigación. [Sitio de internet]. Disponible en: https://bit.ly/3tZuO6i 
Las Drags Queen que participaron son del sexo masculino, con edad de 24 a 29 años y dedicados, dos de ellas, al arte del Drag Queen y una de ellas estudia la licenciatura en Psicología en la UNAM y hace 4 veces al mes, shows de Drag Queen en clubs nocturnos. Las tres viven con sus familias de origen (padre, madre y hermanos/as).

Se presenta en el siguiente esquema, una vez realizada la transcripción de las grabaciones, sólo la tercera parte de la entrevista relacionado con la violencia hacia las mujeres. Las categorías y subcategorías de análisis encontradas son las siguientes.

Figura 2. Esquema de categorías y subcategorías

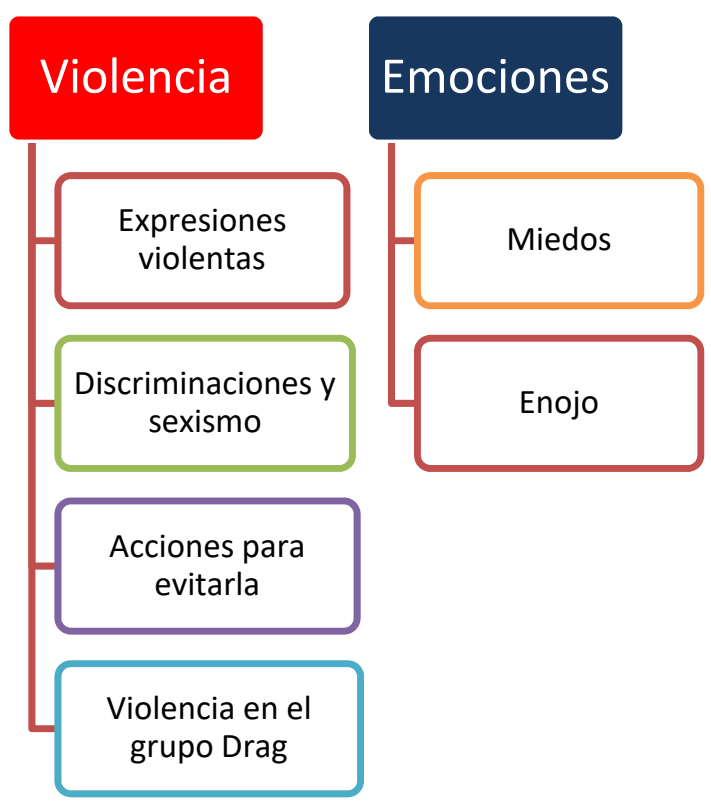

Se presentan las categorías y subcategorías resultado de las entrevistas. Fuente: Autoría propia.

La figura 2 muestra las dos categorías de análisis que se identificaron, una relacionada con las expresiones de la violencia con cuatro subcategorías: expresiones violentas, discriminaciones y sexismo, acciones para evitarla y violencia en el grupo Drag, y, la categoría sobre las emociones en torno a esta violencia, dividido en dos subcategorías: miedos y enojo.

A continuación, se describen los resultados de cada subcategoría.

\section{Subcategoría 1. Expresiones violentas}

Esta primera subcategoría hace referencia a las experiencias violentas que han tenido como Drag Queen, mencionando al insulto a su cuerpo o a meterse con su orientación sexual al decirles "jotitos" o "putitos". Se sienten cosificadas como cuerpos sexuados que pueden ser tocadas sin permiso y las agreden verbalmente dentro de la cotidianeidad en su ámbito laboral o social, estando expuestas constantemente a los peligros diarios que cualquier mujer puede tener. Dentro de las experiencias violentas podemos mencionar las siguientes:

Justo la semana pasada me acaban de hacer algo muy feo, en donde vivo, en Ecatepec, estaba esperando mi combi para venir a la ciudad y arreglarme para una fiesta y traía el pelo largo porque me lo iba a recoger, y pasa un auto gritándome peladeces, como "hay mami y ya sabes que rica y cosas asi" les comentaba a mis amigos que hacía mucho que no me pasaba esa discriminación tan explícita, ya después lo vas sobrellevando y entiendo que la gente lo hace por ignorancia. Hydra. 
En Mérida, me puse un vestido, me arreglé, salí a prender un cigarro, se me hizo super fácil acercarme a una bolita de enfrente de la calle para pedir un encendedor, bueno me vieron como lo peor, como ¿Tú por qué estás aquí?, me dijeron ¿Cómo, qué encendedor? Tú vete de aquí... sentí que no podía transitar por la calle libremente, y ni se lo dije a mis amigos, porque aparte de que se va hacer un pleito enorme, creo que, como drag, aprendes qué batallas vas a librar. También en los baños pasa, a veces pienso en los baños mixtos, pero cuáles baños mixtos mamacita, es una necesidad básica, entonces ¿entro al baño de hombres? Y salgo como mujer, o ¿entro al baño de mujer y salgo como hombre? Pues, se van a sacar mucho de onda, entonces voy a los baños mixtos y trato de cuidarme, sí yo no me cuido nadie lo va hacer por mí. Columba.

Es que estoy normalizando la discriminación, pero pues sí, si lo he pasado, recuerdo bien, viene a mi mente, fui a trabajar a un bar, fui a la barra a pedir un trago y había un tipo, evidentemente heterosexual, y pues yo al final estoy trabajando, estoy hosteando, entonces, yo volteo y le sonreí, y él interpretó mi sonrisa como una carta abierta para faltarme al respeto y cuando yo estoy pidiendo mi trago, él me agarra la nalga. Mi primera reacción fue voltear encabronado y con un léxico bastante florido, le dije ¿Qué chingados te pasa? ¿Estás pendejo o qué? Vuelves hacer eso y te voy mandar a sacar de aquí. Entonces regresé mi mirada con el bar tender y el tipo algo me quiso decir, y yo le puse la mano en la cara y le dije "cállate" y entonces me fui ya de la barra y les dije bien enojado a mis amigos, "un cabrón me hizo esto", entonces me dijeron "güey, ¿pero por qué hiciste eso? que tal que te hace algo", y yo dije, "güey que me haga lo que quiera" no por miedo a que me haga algo, yo no me voy a defender, al final que le da derecho al pendejo, nada más porque uno le sonríe, y me hace pensar en mi mamá, mi hermana, si yo veo que les hacen eso, me le voy encima, creo y espero que el tipo haya aprendido la lección. Andrómeda.

Los discursos anteriores visualizan la violencia hacia la mujer drag no diferenciada de las mujeres cisgénero, sin embargo, Hydra y Columba narran su vivencia desde la expresión femenina donde se reconocen como mujeres en el momento de la agresión, y, por el contrario, Andrómeda, fue la única que narró la historia desde su identidad masculina, presentando una respuesta más agresiva ante la violencia sufrida. Asimismo, en los dos primeros relatos, ambas participantes se encontraban en la calle, lo que las hace mayormente vulnerables a ser agredidas, mientras que Andrómeda se encuentra en su lugar de trabajo con sus compañeras y personal de seguridad que constantemente las protegen, lo que le permite tener una forma distinta para reaccionar ante el evento violento. Olalla y Toala (2020:1) en su trabajo con mujeres violentadas ecuatorianas, reconoce que las redes de apoyo sirven como productoras de resiliencia, siendo las redes informales las más importantes, ya que contar con una persona cercana, sea familiar o amistad, logra que la víctima pueda denunciar el hecho y plantear una salida o trazar nuevos objetivos de vida.

\section{Subcategoría 2. Discriminaciones y sexismo}

Sobre esta categoría de discriminaciones y conductas sexistas que han tenido las participantes, mencionan a los piropos agresivos que experimentan cuando van en la calle, estar expuestas a estar con hombres machistas, alcoholizados y hasta drogados en los bares nocturnos donde trabajan, el tener problemas para usar los baños públicos donde no pueden entrar al de las mujeres por ser hombres ni tampoco al de los hombres porque van vestidos de mujer, y a las burlas y agresiones verbales al caminar, subir a un taxi o llegar a un lugar público. Dentro de sus discursos están:

La gente nos tiene miedo cuando nos ven en la calle y piensan que nos estamos burlando de las mujeres, pero no es así, esto es un desconocimiento de lo que realmente somos y esa ignorancia hace que nos violenten. Hydra.

Las personas se sienten con el derecho a agredirte, porque nos ven diferentes, siendo una expresión de violencia a la diferencia. Columba 
Se normaliza la discriminación de género como parte de nuestra vida, ya que estamos expuestas a esto, al ser diferentes y no queremos entrar en el estereotipo que socialmente nos han impuesto. Andrómeda

Las prácticas discriminatorias y sexistas han traspasado el sexo, el género y las identidades de los seres humanos. En lo referente a las mujeres, su condición de género es por sí mismo una discriminación, no siendo una experiencia igual para todas las mujeres, ya que la interseccionalidad relacionada con la edad, clase, etnia, condición de salud, y expresión sexual hará que se incrementen las prácticas violentas para unas más que para otras. Esto remite a las identidades sociales y contextos históricos y culturales que desarrollan relaciones de poder desigual entre mujeres, produciendo prácticas excluyentes y de estigma social, como las que pueden estar sujetas aquellas mujeres que no cubren con lo socialmente impuesto para pertenecer a lo que se dice llamar "mujer" (Melgar, 2012).

\section{Subcategoría 3. Acciones para evitarla}

Dentro de las acciones que realizan para evitar o frenar la violencia están, desde insultar y gritar fuertemente, hasta golpear a alguien si se acerca más de lo debido. Todas comentan que utilizan su yo masculino para protegerse, aunque reacciona la expresión de mujer que tienen en ese momento, pero actúa el ser masculino que llevan dentro. Esta acción no lo consideran violencia sino una forma de defensa o protección.

Otro discurso que comentan es el apoyo social del grupo drags, siendo sus guardaespaldas, cuidándose constantemente entre ellas y socializando lo que les pasa para evitar que a otras les suceda. Columba menciona "hago un check-in cuando voy al baño y cuido a mis compañeras cuando ellas van, nos echamos a correr todas si estamos en peligro".

\section{Subcategoría 4. Expresiones de violencia en el grupo Drag}

Esta subcategoría resulta interesante, debido a que, dentro de la cotidianeidad en los grupos drags, se encuentra el uso del lenguaje grotesco entre ellas, por lo que era importante conocer qué pensaban al respecto del uso de palabras como "perras", "putas", "zorras", etc.

Las respuestas de las tres participantes hacen referencia a una sobreprotección o empoderamiento al utilizar estas palabras entre ellas. Mencionan que sólo si eres parte del grupo drags puedes decirlas sin sentirse ofendidas, siendo un insulto fuera del grupo. Para Andrómeda, es una forma de "apoderarse de los estigmas para desarmar al estigmatizador", de tal manera, que le quita el significado violento en lo habitual y deja de ser una grosería, para formar parte de las prácticas relacionales en exclusividad al grupo y de la misma identidad de la comunidad Drag.

El lenguaje es una expresión simbólica de la experiencia del individuo que permite visibilizar la realidad que nos rodea; nos permite delinearla, concretizarla y habilitarla para reflexionar sobre ella y llevarla al escenario de la controversia, aceptación, diferencia y diversidad. Por ello, se fundamenta desde la identidad y nos cobija en la pertenencia al grupo social en el que vivimos (Sánchez, 2014:2).

Haciendo un resumen de esta primera categoría, retomamos los ejes de análisis que Hernández y Recalde (2020) mencionan sobre los espacios públicos, donde las formas de regulación sexogenéricas se articulan y reproducen de manera diferente que como se viven en los espacios privados. El cuerpo, el género y la sexualidad son constructos espaciados, que conllevan experiencias de placer, resistencia y resignificación. Las expresiones violentas y de discriminación que las drags entrevistadas compartieron, dan cuenta de los discursos, prácticas y representaciones sociales de las nuevas sexualidades y de cómo quebrantan lo social establecido ante las lógicas hegemónicas para vincularse a un cuerpo emancipado, fluido y transgresor.

Revista del Laboratorio Iberoamericano para el Estudio Sociohistórico de las Sexualidades https://doi.org/10.46661/relies.6288 
Para la segunda categoría de resultados, relacionada con las expresiones emocionales, se encontraron dos subcategorías.

\section{Subcategoría 1. Miedos}

Las respuestas de miedo que las participantes mencionan son diferentes en ellas. Mientras que, para Hydra menciona el tener miedo a perder a su familia, a estar sola y no tener amistades o a la muerte de un ser querido, para Columna presenta miedo a que la maten cuando va vestida en la calle como drag o la lastimen en su cuerpo o hasta la desaparezcan, ya que ha tenido compañeras a las que les ha pasado esto. Y, similarmente para Andrómeda también es miedo a que la maten o la golpeen o agredan sexualmente, confrontar a la gente que puede asediarla en la calle le provoca miedo y a la vez enojo.

En las tres participantes, la emoción del miedo se ve influenciado por la construcción del estereotipo tradicional femenino y las violencias que se ejercen hacia las mujeres.

Soto (2013:203) menciona que el concepto de género muestra a la emoción de miedo y las expresiones violentas dentro de espacios públicos en vinculación diferenciada, donde las mujeres presentan más miedo a ser agredidas sexualmente y maltratadas en sus cuerpos que los hombres. Las emociones de miedo son expresiones subjetivas del pensamiento binario, que colocan a la mujer en relaciones de poder desigual influidas por las prácticas de socialización de éstas en vulnerabilidad contextual; es decir, como productos culturales reproducidos por experiencias de corporeidad.

\section{Subcategoría 2. Enojo}

Las emociones que sienten cuando se encuentran con alguna experiencia violenta como mujeres drag, les provoca enojo en las tres entrevistadas, siendo para Hydra, además, un sentimiento de coraje por estar viviendo esa violencia. Comentan que se sienten vulneradas y que reaccionan en función del contexto, ya que saben que están vestidas como mujeres y la ropa, los tacones y demás accesorios no les permiten actuar fácilmente para defenderse como cuando visten de hombres. Saber que representan a una mujer y que eso las hace sentir vulnerables también les provoca enojo.

Las respuestas de las participantes están vinculadas en su condición sexual masculina, ya que estructuran la violencia y sus emociones en torno al enojo y coraje y no desde el miedo, que las mujeres sienten cuando son violentadas. Esta diferencia habla de la emoción como práctica ideológica y civilizatoria que establece la contextualización de las emociones en la vida social. En este sentido, las emociones se asocian a las mujeres de acuerdo a los sistemas de creencias y de relaciones de poder vinculadas al género, lo que no les permite sentir enojo y coraje sino miedo e impotencia (Illouz, 2007).

En resumen, en esta categoría vemos que las emociones que experimentan las entrevistadas se ubican en espacios públicos, donde el imaginario social pone en cuestión el comportamiento, la manera de vestir y la expresión del cuerpo, mediados por emociones como culpa, miedo, intimidación e inseguridad. Robles (2014) menciona que el miedo en las mujeres está estrechamente asociado con las percepciones de las personas que ocupan el espacio público y que, a la vez, lo controlan, “...las condiciones sociales son las productoras de los miedos de las personas como parte de los hechos violentos a los cuales nos enfrentamos día con día; creando imaginarios sociales de inseguridad y desconfianza del otro, y por tanto reforzando las desigualdades entre los seres humanos... (pág. 98)". Refiere, que la emoción de miedo en espacios públicos, está vinculado a diferencias de género, donde los hombres construyen la emoción hacia el miedo a perder sus 
posesiones o bienes, o a perder su vida, mientras que las mujeres lo elaboran desde la posesión de un cuerpo sexuado y valorado socialmente desde esa Otredad existente en dicho espacio (Robles, 2014).

\section{Conclusiones}

A modo de conclusión podemos decir que la principal emoción que reportan las entrevistadas es el miedo a ser agredidas en sus cuerpos cuando se expresan como mujeres drag, combinado con el enojo al permitirlo. Mencionan que deben limitar el querer golpear a la persona que las agrede debido a que adoptan una postura femenina donde "saben" que no "deben" expresar conductas agresivas adoptando conductas estereotipadas al género femenino. Sin embargo, quedan impotentes para contestar "como hombres". Buscan el apoyo social cuando sucede y si están solas analizan si pueden defenderse como hombres (si es un solo agresor) o deben huir (si son más de dos agresores), por miedo a ser lastimadas o a ser asesinadas (homicidio de odio). Analizan el lugar donde se encuentran y miden el riesgo para saber qué acciones deben tomar frente a las conductas violentas. También hacen uso de sus redes de apoyo para sentirse seguras, principalmente en la comunidad drag o con familiares y amistades.

El estudio de las emociones en torno a la violencia en las expresiones de ser mujer no varía para una Drag Queen o para una mujer Cis, ya que sigue siendo una expresión de desigualdad de poder. Se requiere de propuestas de políticas públicas en torno a la prevención, atención y disminución de la violencia hacia las mujeres, en pro de proteger el derecho al libre ejercicio de las sexualidades tanto en las mujeres y niñas como para las demás expresiones femeninas.

\section{Referencias}

Acién, E. \& Checa, F. (2020). Estigma, políticas públicas y violencia. Discursos de trabajadoras sexuales activistas sobre la violencia institucional en Argentina y España. Revista del Laboratorio Iberoamericano para el Estudio Sociohistórico de las Sexualidades, 4(01), 07-36. https://www.upo.es/revistas/index.php/relies/article/view/4793

Álvarez-Gayou J.L. (2006). Cómo hacer investigación cualitativa. Fundamentos y metodología. Paidós Educador; 187-190.

Bernini, L. (2017) Ejercicio de la crítica queer. ¿Cómo funciona la sexualidad? En L. Bernini. Las teorías queer. Una introducción. (pp. 49-95). Egales.

Butler, J. (1990) Gender Trouble: Feminism and the Subversion of Identity. (pp. 20-29) Routledge.

Butler, J. (1993) Bodies that matter. On the discursive limits of sex. (223-242) Routledge.

Butler, J. (2001) El género en disputa. El feminismo y la subversión de la identidad. Paidós/PUEG UNAM.

Cepeda, M., \& Flores, X. (2011) Terrorismo de género. Aproximaciones al movimiento Drag en Lima. Anthropia, 9, 16-27 http://revistas.pucp.edu.pe/index.php/anthropia/article/view/11241/11752

CNCLVD (2014) Violencia basada en género y generaciones: una mirada desde la comunicación. Uruguay: Consejo Nacional Consultivo de Lucha contra la Violencia Doméstica. Sistema Integral de 
Protección a la Infancia y Adolescencia contra la Violencia. Género 1 Ministerio de Desarrollo Social (www.gub.uy)

Córdova, P. (2011) Sexualidades Disidentes: entre los cuerpos normatizados y cuerpos lábiles. Revista de estudios de género, 4(33), 42-72. http://www.scielo.org.mx/scielo.php?script=sci_arttext\&pid=S14054362011000100004\&lng=es\&t $\underline{\text { lng }=e s}$

Hernández, J.O. y Recalde, L. (2020) Introducción. Espacio público: sexualidad, cuerpo y Género. Prácticas, representaciones y discursos. Revista del Laboratorio Iberoamericano para el Estudio $\begin{array}{llll}\text { Sociohistórico de las 3(01), 01-04. } & \text { Sexualidades, }\end{array}$ https://www.upo.es/revistas/index.php/relies/article/view/4900

Hernández, R., Fernández, C. \& Baptista, P. (2018) Metodología de la investigación: Las rutas cuantitativa, cualitativa y mixta. Mac Graw Hill; pp. 524.

Illouz, E. (2010). Redes románticas. En E. Illouz, Intimidades congeladas. (pp. 161-226). Katz

Lamas, M. (2016). Género. En H. Moreno \& E. Alcántara (Coord.). Conceptos clave de los estudios de género. Vol.1 (pp. 155-186). Centro de Investigaciones y Estudios Interdisciplinarios, UNAM.

Maffia, D. \& Cabral, M. (2003) ¿Los sexos son o se hacen? En D. Maffia (Comp.). Sexualidades migrantes. Género y transgénero. (pp. 86-96). Feminaria. http://dianamaffia.com.ar/archivos/sexualidadesmigrantesdm.pdf

Melgar, L. (2012) Discriminación sobre discriminación: una mirada desde la perspectiva de género. Colección Equidad de género y democracia, vol. 5; - México: Suprema Corte de Justicia de la Nación, Tribunal Electoral del Poder Judicial de la Federación, Instituto Electoral del Distrito Federal.

Moncrieff, M. \& Lienard, P. (2017) A natural History of the Drag Queen Phenomenon. Evolutionary Psychology. Abril-junio 1-14 DOI 10.1177/1474704917707591

Moreno, T. \& Torres, C. (2018) Performatividad. En H. Moreno \& E. Alcántara (Coord.). Conceptos clave de los estudios de género Vol. 2 (pp. 233-250). Centro de Investigaciones y Estudios Interdisciplinarios, UNAM.

Olalla, J. \& Toala, K. (2020). Redes de apoyo en mujeres víctimas de violencia, una propuesta para generar resiliencia. Revista Desarrollo y Transformación Social. 1-10. Universidad Internacional SEK.https://repositorio.uisek.edu.ec/bitstream/123456789/3780/4/Art\%C3\%ADculo\%200lalla\%20 Naranjo\%20Jenny\%20\%20Toala\%20Zavala\%20Kevin\%C2\%A0.pdf

Pérez, K. (2011). Historia de la patologización y despatologización de las variantes de género. En M. Missé \& G. Coll-Planas (Coord.). El género desordenado, críticas en torno a la patologización de la transexualidad. (pp. 97-115) Egales.

Pons, R. \& Garosi, E. (2016). Trans. En H. Moreno \& E. Alcántara (Coord.). Conceptos clave de los estudios de género. Vol.1. (pp. 307-327). Centro de Investigaciones y Estudios Interdisciplinarios, UNAM. 
Recalde, B. \& Gómez, S. (2016). Entre ficciones y subversiones. Repensando los cuerpos sexuados y generalizados. En E. Celisto, S. Gómez, E. Grabino, A. Magnone, B. Recalde, D. Rostagnol \& C. Viera. Trashumancias Búsqueda teóricas feministas sobre cuerpo y sexualidad. (pp 39-58) CSIC, Universidad de la República Uruguay.

Robles, A.L. (2014). Miedo en las calles: principal emoción de la inseguridad pública delictiva. Un estudio criminológico y de género. Revista IUS, 8(34), 81-100. http://www.scielo.org.mx/scielo.php?script=sci arttext\&pid=S1870-21472014000200006

Salinas, L. (1994) La construcción social del cuerpo. Reis Revista Española de Investigaciones Sociolingüísticas. (68) 85-96. http://www.reis.cis.es/REIS/PDF/REIS_068_06.pdf

Sánchez, G. (2014) Identidades de Género y Sexuales en el Lenguaje. Lenguaje Políticamente Correcto: Dos Caras de la Moneda. (1-20) V Jornadas de Graduados-Jóvenes Investigadores FaHCEUNLP. http://www.memoria.fahce.unlp.edu.ar/trab eventos/ev.4088/ev.4088.pdf

Soto Villagrán, P. (2013). Entre los espacios del miedo y los espacios de la violencia: discursos y prácticas sobre la corporalidad $y$ las emociones. En M. A. Aguilar y P. Soto (Coord.), Cuerpos, espacios y emociones. Aproximaciones desde las ciencias sociales (197-219). UAM.

Strübel-Scheiner, J. (2011). Gender Performativity and Self-Perception: Drag as Masquerade. International Journal of Humanities and Social Science, 1(13), 12-19 http://ijhssnet.com/journals/Vol 1 No 13 Special Issue September 2011/2.pdf

Toquero, B. (2018). El surgimiento de las Drag Queen, una forma de expresión que se populariza entre la comunidad LGBT. Epikeia 38(1) pp. 1-12 https://doctrina.vlex.com.mx/vid/surgimientodrag-queen-forma-706707573

Villanueva, J. (2017). "Yo soy una drag Queen, no soy cualquier loco". Representaciones del dragqueenismo en Lima, Perú. Península, 12(2), 95118. https://doi.org/10.1016/i.pnsla.2017.06.005 